\title{
Ocular lubricant use in medically and surgically treated glaucoma: a retrospective longitudinal analysis
}

This article was published in the following Dove Press journal:

Clinical Ophthalmology

23 June 2017

Number of times this article has been viewed

Jayant Venkatramani lyer ${ }^{1-3}$

Yang Zhao ${ }^{3}$

Fiona Pin Miao Lim ${ }^{1,2}$

Louis Tong ${ }^{1-4}$

Tina Tzee Ling Wong ${ }^{1-4}$

'Glaucoma, Singapore National

Eye Centre, ${ }^{2}$ Singapore Eye

Research Institute, ${ }^{3}$ Department of Ophthalmology, Yong Loo Lin School of Medicine, National University of

Singapore, ${ }^{4}$ Ophthalmology Academic

Clinical Program, Duke-NUS Graduate

Medical School, Singapore
Correspondence: Tina Tzee Ling Wong Singapore National Eye Centre, II Third Hospital Avenue, Singapore 16875I,

Singapore

Tel +6562277255

Fax +65 62263995

Email tina.wong.t.l@singhealth.com.sg
Background: Chronic use of intraocular pressure-lowering medications is associated with ocular surface disease (OSD). In this study, we assess the incidence of topical lubricant use as a surrogate marker for underlying OSD, in medically and surgically treated glaucoma patients.

Methods: Retrospective chart review was performed for newly diagnosed glaucoma patients who were started on topical medications in 2007 and followed up over a 5-year period. Primary outcome measure was the incidence of topical lubricant use in these patients and a subset of these patients who required glaucoma or cataract surgery during follow-up.

Results: Charts of 505 newly diagnosed glaucoma patients with no prior history of ocular lubricant use were analyzed. Mean age was 63.9 years (SD 11.1), 42.8\% were women. One hundred one $(20.0 \%)$ patients underwent phacoemulsification surgery, 80 underwent mitomycin $\mathrm{C}$ (MMC) augmented phacotrabeculectomy, 16 underwent MMC-augmented trabeculectomy and 3 underwent tube surgery during the course of follow-up as their only type of surgery. Five-year incidence of lubricant use was 59\% in all glaucoma subjects; $54.1 \%$ of patients were on medical treatment and $74.0 \%$ of patients who underwent phacotrabeculectomy or trabeculectomy were started on lubricants, respectively $(P=0.0011) ; 60.4 \%$ of glaucoma subjects who underwent phacoemulsification surgery were started on lubricants. Incidence of lubricant use increased from $17.7 \%$ preoperatively to $74.0 \%$ postoperatively in subjects who required trabeculectomy or phacotrabeculectomy. Incidence of lubricant use was similar in patients on one or multiple glaucoma medications, with the mean onset of lubricant use being 10 months after starting glaucoma medication in both groups. Females were more likely to use artificial tears compared with males $(P=0.002)$.

Conclusion: Both medical and surgical management of glaucoma have an adverse effect on the ocular surface. Chronic use of glaucoma medications was associated with a high incidence of ocular lubricant use. MMC-augmented trabeculectomy was associated with an even higher incidence of ocular lubricant use.

Keywords: glaucoma, medications, trabeculectomy, ocular surface disease, ocular lubricants

\section{Introduction}

Glaucoma is one of the leading causes of irreversible blindness worldwide. ${ }^{1}$ The global prevalence of glaucoma in a population aged $40-80$ years is $3.54 \%$ and the number of people with glaucoma worldwide is expected to increase to 111.8 million by $2040 .^{2}$ Treatment of glaucoma is mainly targeted at lowering intraocular pressure (IOP) using medication or surgery.

Patients with glaucoma have been shown to suffer from ocular surface disease (OSD) at a higher prevalence rate than patients without glaucoma. ${ }^{3-11}$ This has been largely attributed 
to chronic use of topical IOP-lowering medications in this population..$^{3-11}$ Topical ocular IOP-lowering drugs are believed to trigger or exacerbate OSD by inducing ocular surface damage, especially if they contain preservatives, such as benzalkonium chloride (BAK). ${ }^{12}$ Preservatives in eye drops have been shown to be toxic to the ocular surface epithelium and decrease tear film stability. ${ }^{8,9}$ Topical glaucoma medications have also been associated with alterations in Meibomian gland function and morphology, ${ }^{3}$ tear film instability, ${ }^{10-13}$ decreased tear turnover and tear production, ${ }^{14-16}$ corneal anesthesia, ${ }^{17,18}$ corneal epithelial barrier dysfunction ${ }^{19,20}$ and inflammatory changes in the conjunctiva, ultimately resulting in OSD. ${ }^{21-24}$

Few studies have examined the impact of glaucoma surgery on the ocular surface. In fact, intolerance to topical medications resulting in conditions such as chronic conjunctivitis is an indication for glaucoma surgery. ${ }^{25}$ Lee et al revealed greater tear film osmolality and dry eye symptoms in both medically and surgically treated glaucoma patients. ${ }^{26}$ Neves Mendes et al demonstrated that filtering blebs directly interfered with tear film stability - with demonstrable interference to the tear breakup time (TBUT) and Schirmer test - regardless of the bleb's height and extent. ${ }^{27}$ Dry eye symptoms were not assessed in this study. ${ }^{27} \mathrm{~A}$ more recent study by Lam et al described a retrospective case series of patients with OSD following trabeculectomy and found reduced TBUT and Schirmer scores in this group of patients. ${ }^{28}$ This study also found that the treatment of OSD with lubricants improved best corrected visual acuity in $50 \%$ of these subjects. ${ }^{28}$

OSD is a multifactorial disease involving tears and ocular surface that may present clinically with symptoms of dry eyes, including gritty sensation and transient blurring of vision, and may be accompanied by signs of reduced TBUT or a lower Schirmer score. ${ }^{29}$ However, clinical indicators of OSD may not necessarily correlate well with ocular symptoms of OSD. ${ }^{30-32}$ In fact, it has been previously reported that only $57 \%$ of subjects with dry eye symptoms actually had clinical signs such as reduced TBUT or Schirmer score. ${ }^{33}$ Symptom assessment used on its own is also very subjective. A dependence on topical lubricants may be used as a surrogate marker for underlying OSD.

In our study, we examine the incidence of ocular lubricant use in glaucoma patients over 5 years after commencing topical IOP-lowering medication. In addition, we assessed ocular lubricant use in a subset of this group of patients who subsequently required glaucoma filtration surgery during the course of follow-up. This is the first study to our knowledge to assess incidence of ocular lubricant use in a large number of medically and surgically treated glaucoma patients.

\section{Methods \\ Study design}

Our study was a retrospective, noninterventional, longitudinal chart review, which was conducted in a single institution (Singapore National Eye Centre). It followed the principles of the Declaration of Helsinki. Ethical approval was obtained from the Singhealth Centralized Institutional Review Board (CIRB Reference number 2012/484/A). Patient consent was not required to review their medical charts, as this was an anonymized retrospective chart review. Patient confidentiality was ensured during handling of data.

\section{Study recruitment}

Medical and pharmaceutical records of patients who were diagnosed with glaucoma (primary and secondary) or ocular hypertension and commenced on topical IOP-lowering therapy in 2007 were reviewed. Six hundred and ninetyseven patients with glaucoma medications started in 2007 and no usage of artificial tears prior to starting glaucoma medication were identified based on prescription records and reviewed.

\section{Inclusion and exclusion criteria}

Only adult patients $>35$ years of age diagnosed with glaucoma and with no prior record-based use of topical lubricants were recruited. Exclusion criteria included the presence of conditions that could compromise the ocular surface including preexisting thyroid eye disease, rheumatoid arthritis, pterygium, systemic lupus erythematosus, trichiasis, ectropion, entropion, Bell palsy or recent (within 6 months) ocular surgery.

\section{Data collection, surgery and primary outcome}

Data on patient demographics, type and number of topical IOP-lowering eye drops, artificial tears usage incidence up to 5 years after commencement of IOP-lowering eye drops were collected and analyzed. In addition, the number of patients requiring intraocular surgery - phacoemulsification, mitomycin $\mathrm{C}(\mathrm{MMC})$ augmented trabeculectomy and $\mathrm{MMC}$ augmented phacotrabeculectomy - was also analyzed.

MMC-augmented trabeculectomy in our center is performed using a fornix-based conjunctival flap. MMC is topically applied subconjunctivally using soaked sponges at a concentration of $0.4 \mathrm{mg} / \mathrm{mL}$ for a typical duration of 2 minutes, following which a washout is performed with 20-50 mL of balanced salt solution. Trapezoid scleral flaps are fashioned prior to sclerostomy and peripheral iridectomy. 
Nylon 10-0 sutures are used to close the scleral flaps and conjunctiva.

The primary outcome measure was the proportion of patients who required prescription of artificial tears during the 5-year follow-up period.

\section{Statistical analysis}

Differences between groups were tested using the independent $t$-test and chi-square test. For the survival analysis, the primary end point was the time to the onset of artificial tears use. A $P$-value of $<0.05$ was considered to be statistically significant. All analyses were performed using SPSS Version 21 (IBM Corporation, Armonk, NY, USA).

\section{Availability of data and materials}

All data generated or analyzed during this study are included in this published article.

\section{Results}

Of the 697 records reviewed, 121 were found not to have glaucoma or ocular hypertension and were excluded from the study. Of the remaining 576 patients, 71 were excluded as they had pre-existing conditions (most common causes: recent ocular surgery, pterygium, lid disease and autoimmune conditions) that could aggravate OSD. A total of 505 patients were, therefore, included in our study with a mean follow-up duration of 59.3 months (SE 3.90). Of the 505 patients, 25 patients were lost to follow-up before 5 years with a mean duration of follow-up of 46.8 months (SE 12.1). The distribution of glaucoma types may be found in Table 1.

\section{Demographics}

About $57 \%$ of the glaucoma subjects were male. Eighty-two percent, $6 \%$ and $8 \%$ of the subjects were of Chinese, Malay and Indian descent, respectively. Mean age of the subjects was 63.9 years (SD 11.1) (Tables 1 and 2).

During the 5 years of review, $124(24.6 \%)$ glaucoma subjects underwent cataract surgery (phacoemulsification), 25 underwent trabeculectomy with MMC, 97 underwent phacotrabeculectomy with MMC and 3 underwent tube shunt surgery. Some of these patients may have undergone multiple procedures in either eye, like a phacoemulsification followed by trabeculectomy at another setting. For subsequent comparative analysis between surgical procedures, only patients who underwent single type of surgery were included (101 phacoemulsifications, 16 trabeculectomies and 80 phacotrabeculectomies). Two hundred twenty-two patients had no surgery at all during the 5-year period.
Table I Patient demographics

\begin{tabular}{ll}
\hline Parameters & Mean (SE), N (\%) \\
\hline Age (years) & $63.89(0.49)$ \\
Race & \\
Chinese & $414(82.0)$ \\
Malay & $32(6.3)$ \\
Indian & $42(8.3)$ \\
Others & $17(3.4)$ \\
Gender & \\
Male & $289(57.2)$ \\
Female & $216(42.8)$ \\
Glaucoma type & \\
POAG & $191(37.8)$ \\
PACG & $115(22.8)$ \\
NTG & $67(13.3)$ \\
Secondary & $48(9.5)$ \\
Neovascular & $16(3.2)$ \\
Pseudophakic & $11(2.2)$ \\
Pseudoexfoliative & $8(1.6)$ \\
Traumatic & $6(1.2)$ \\
Pigment dispersion syndrome & $3(0.6)$ \\
Uveitic & $3(0.6)$ \\
PSS & $2(0.4)$ \\
Silicone oil & $2(0.4)$ \\
Angle recession & $1(0.2)$ \\
Unspecified & $2(0.4)$ \\
Not specified & $6(1.2)$ \\
OHT & $49(9.7)$ \\
PAC & $29(5.8)$ \\
\hline
\end{tabular}

Abbreviations: NTG, normal tension glaucoma; $\mathrm{OHT}$, ocular hypertension; PAC, primary angle closure; PACG, primary angle closure glaucoma; POAG, primary open angle glaucoma; PSS, Posner-Schlossman syndrome.

\section{Eye drop usage}

The topical IOP-lowering eye drops used by the glaucoma patients included latanoprost, bimatoprost, travoprost, betaxolol, timolol, brinzolamide, dorzolamide, pilocarpine and brimonidine as well as combinations of the above. Only 3 patients used preservative-free Timabak ${ }^{\circledR}$, whereas the rest used IOP-lowering eye drops with preservatives.

Table 2 Patients who underwent surgery* over 5 years of review

\begin{tabular}{ll}
\hline & Mean, N (\%) \\
\hline All intraocular surgery & $283(56.0)$ \\
Cataract surgery & $124(24.6)$ \\
Phacoemulsification as only surgery & $101(20.0)$ \\
Tr & $25(5.0)$ \\
$\quad$ Tr as only surgery & $16(3.2)$ \\
PT & $97(19.2)$ \\
PT as only surgery & $80(15.8)$ \\
Tube-shunt surgery & $3(0.6)$ \\
\hline
\end{tabular}

Notes: *Some patients underwent a combination of different types of surgery during the 5-year review. "The number of patients who underwent only one type of surgery throughout the 5 years.

Abbreviations: MMC, mitomycin C; PT, MMC-augmented phacotrabeculectomy; $\operatorname{Tr}$, MMC-augmented trabeculectomy. 
Table 3 Lubricant use in glaucoma patients

\begin{tabular}{ll}
\hline Lubricant use & $\mathbf{N}(\%)$ \\
\hline All cases $(\mathrm{n}=505)$ & $298(59.0)$ \\
No surgery over 5 years $(\mathrm{n}=222)$ & $120(54.1)$ \\
Tr alone or PT alone $(\mathrm{n}=96)$ & $7 \mathrm{I}(74.0)$ \\
PE alone $(\mathrm{n}=10 \mathrm{I})$ & $61(60.4)$ \\
\hline
\end{tabular}

Abbreviations: MMC, mitomycin C; PE, phacoemulsification; PT, MMC-augmented phacotrabeculectomy; Tr, MMC-augmented trabeculectomy.

The topical artificial tears used in our study included Hypromellose 0.3\% (EyeMO, GlaxoSmithKline, Philadelphia, PA, USA), Tears Naturale Preservative Free (Alcon Laboratories Inc, Fort Worth, TX, USA), Duratears eye ointment (Alcon Laboratories Inc), Vidisic eye gel (Bausch and Lomb, Surrey, UK), Systane Ultra (Alcon Laboratories Inc) and Thera Tears (Akorn Pharmaceuticals, Decatur, IL, USA).

\section{Lubricant usage}

Over 5 years, $298(59.0 \%)$ of the 505 glaucoma subjects were started on topical lubricants. Two hundred twenty-two subjects did not undergo any intraocular surgery over 5 years of follow-up; 120 of these patients (54.1\%) were started on ocular lubricants during this period (Tables 3 and 4; Figure 1).

A significantly higher proportion of patients were started on lubricants in the post-trabeculectomy group (including phacotrabeculectomy and trabeculectomy) $(74.0 \%)$ than in the medically treated group (54.1\%) $(P=0.0011)$.

Of the 80 glaucoma patients who underwent phacotrabeculectomy, the proportion of subjects using lubricants increased from $17.5 \%$ preoperatively to $76.3 \%$ postoperatively. Of the 16 glaucoma patients who underwent plain trabeculectomy, the proportion using ocular lubricants increased from $18.8 \%$ preoperatively to $65.4 \%$ postoperatively. Pooling the subjects who underwent phacotrabeculectomy and trabeculectomy, 71 (74.0\%) subjects were prescribed lubricants postoperatively (mean onset to lubricant use: 12.15 months, SD: 13.52) compared with just 17 of them (17.7\%) preoperatively.

In a subgroup of patients who had undergone either trabeculectomy (plain or combined with phacoemulsification) or phacoemulsification, significantly more patients were started on lubricants in the post-trabeculectomy

Table 4 Onset of lubricant use in patients with the same glaucoma medication prescription in the first year of follow-up

\begin{tabular}{lll}
\hline & Monotherapy & $\begin{array}{l}\text { Combination } \\
\text { therapy }\end{array}$ \\
\hline $\begin{array}{l}\text { Number of patients started } \\
\text { on lubricants (\%) }\end{array}$ & $114(56.7)$ & $31(57.4)$ \\
$\begin{array}{l}\text { Mean number of months to } \\
\text { onset of lubricant use (SE) }\end{array}$ & $10.15(0.28)$ & $10.48(0.49)$ \\
\hline
\end{tabular}

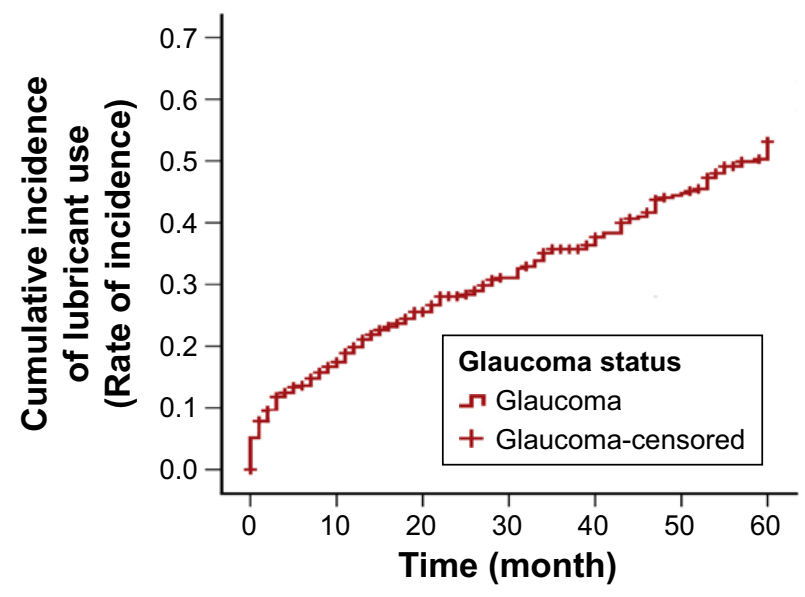

Figure I Cumulative incidence of lubricants.

group $(74.0 \%)$ than in the post-phacoemulsification group $(60.4 \%)(P=0.0495)$.

In the whole cohort, females were more likely to use artificial tears compared with males ( $P=0.002)$. In 255 glaucoma subjects with no medication change over 1 year of follow-up, there was no significant difference in the onset of lubricant use between the patients treated with just one type of medication (monotherapy) and those on combination/multiple therapy $(P=0.928)$. One hundred fourteen patients $(56.7 \%)$ on monotherapy compared with 31 patients $(57.4 \%)$ on combination therapy were started on lubricants during 1 year of follow-up. Mean onset time to lubricant use was 10.15 months (SE 0.28) in patients on monotherapy and 10.48 months (SE 0.49) in patients on combination therapy.

Among 201 glaucoma subjects on monotherapy, there was also no significant difference found in the onset of lubricant use among all the classes of glaucoma medication $(P=0.206)$.

\section{Discussion}

In our study, we have found that a high percentage (54.1\%) of medically treated glaucoma patients required a prescription for ocular lubricants over a 5-year period. In comparison, another French population-based study found that just $14.7 \%$ of elderly subjects required the use of artificial tears. ${ }^{34}$

The findings of our study are consistent with that of numerous studies that implicate topical glaucoma medications in OSD. ${ }^{3-11}$ A recently published prospective study by Saade et al similarly found $68 \%$ of glaucoma patients on topical IOP-lowering drops to have signs suggestive of OSD compared with just $17 \%$ of controls without glaucoma or previous topical therapy. ${ }^{35}$

Another interesting finding in our study was that there was no difference in the incidence of the use of lubricants 
between patients who were on monotherapy - regardless of the choice of agent - and those on multiple/combination IOP-lowering medications. More than half of the patients had a prescription for ocular lubricants regardless of the number or type of topical glaucoma medications used.

Recent studies have found that chronic use of topical IOPlowering eye drops results in alterations in meibomian gland function and morphology, ${ }^{3}$ tear film instability, ${ }^{10-13}$ decreased tear turnover and tear production, ${ }^{14-16}$ corneal anesthesia, ${ }^{17,18}$ corneal epithelial barrier dysfunction ${ }^{19,20}$ and inflammatory changes in the conjunctiva ${ }^{21-24}$ causing OSD. Preservatives in glaucoma drops have been cited as the main culprit - studies have found that these preservatives decrease tear film stability and cause toxicity to the ocular surface epithelium in vitro and in vivo in both clinical and animal studies. ${ }^{8,9}$ As such, some propose the use of preservative-free IOP-lowering eye drops. One paper reported that BAK-free travoprost may even have beneficial effects on the ocular surface. ${ }^{12}$ Too few patients were on preservative-free formulations to analyze and account for the effects of preservatives, in our study.

Although it has been known that glaucoma filtration surgery was also associated with OSD, we were surprised to note a four-fold rise in the use of ocular lubricants postoperatively among glaucoma patients who underwent MMC-augmented trabeculectomy. In addition, we found that trabeculectomy surgery was associated with a greater use of ocular lubricants postoperatively $(74.0 \%)$ compared with phacoemulsification surgery $(60.4 \%)$. One study found ocular surface complications including corneal epitheliopathy, filamentary keratitis and dellen in $15 \%$ of patients following trabeculectomy. ${ }^{36}$ One possible reason for OSD in these patients could be the use of MMC. MMC is an antimetabolite and targets actively replicating cells including corneoscleral limbal cells. This could prevent adequate replacement of the corneal epithelium resulting in OSD. ${ }^{37} \mathrm{~A}$ stable tear film, corneal and conjunctival epithelium in anatomic continuity with the lacrimal glands and eyelids are important in keeping a stable ocular surface. ${ }^{38}$ Anatomical disruption to the normal conjunctival and corneal architecture due to the presence of a bleb following trabeculectomy could be another cause for OSD and the high incidence of ocular lubricant use in this subset of glaucoma patients in our study.

Glaucoma on its own is known to pose a significant economic burden and have a significant impact on health-related quality of life (HRQoL). ${ }^{39}$ Direct costs for the management of glaucoma in the USA alone are estimated to be in the range of $\$ 2.9$ billion per year. ${ }^{40}$ Visual field defects from glaucoma also impose a negative impact on patients' HRQoL and a psychological burden on their families. ${ }^{39}$ The increased incidence of ocular lubricant use in glaucoma patients, as demonstrated in our study, suggests additional economic burden and HRQoL impact.

This is the first large-scale study to our knowledge exploring the functional impact of OSD - using ocular lubricant use as a surrogate marker - in patients with medically and surgically treated glaucoma over a relatively long duration of 5 years. The major limitation of our study is that it is a retrospective case review. Another limitation is that too few of our subjects were on preservative-free IOP-lowering medication to make any meaningful analysis for the effect ocular preservatives may have on incidence of ocular lubricant dependence. We were also unable to account for the frequency of IOP-lowering eye drops in relation to OSD as there were often changes in prescriptions, use of a combination of eye drops and compliance issues over the 5-year follow-up.

\section{Conclusion}

In conclusion, the chronic use of IOP-lowering eye drops is associated with an increased use of lubricant eye drops. There is an even higher incidence of lubricant usage in such patients following MMC-augmented trabeculectomy surgery. Future studies could explore the role of preservative-free IOP-lowering medications in glaucoma subjects in relation to ocular lubricant dependence.

\section{Author contributions}

JVI critically reviewed and analyzed the data in addition to drafting the manuscript for publication. YZ played a key role in gathering data and performing statistical analysis. FPML conducted the retrospective chart review and helped edit the manuscript. TTLW and LT conceived the study, participated in its design and coordination and helped review and edit the manuscript. All authors contributed toward data analysis, drafting and revising the paper and agree to be accountable for all aspects of the work.

\section{Disclosure}

The authors report no conflicts of interest in this work.

\section{References}

1. Bourne RR, Stevens GA, White RA, et al. Causes of vision loss worldwide, 1990-2010: a systematic analysis. Lancet Glob Health. 2013;1(6):339-349

2. Tham YC, Li X, Wong TY, Quigley HA, Aung T, Cheng CY. Global prevalence of glaucoma and projections of glaucoma burden through 2040: a systematic review and meta-analysis. Ophthalmology. 2014; 121(11):2081-2090.

3. Arita R, Itoh K, Maeda S, et al. Comparison of the long-term effects of various topical antiglaucoma medications on meibomian glands. Cornea. 2012;31(11):1229-1234. 
4. Fechtner RD, Godfrey DG, Budenz D, Stewart JA, Stewart WC, Jasek MC. Prevalence of ocular surface complaints in patients with glaucoma using topical intraocular pressure-lowering medications. Cornea. 2010;29(6):618-621.

5. Ghosh S, O’Hare F, Lamoureux E, Vajpayee RB, Crowston JG. Prevalence of signs and symptoms of ocular surface disease in individuals treated and not treated with glaucoma medication. Clin Experiment Ophthalmol. 2012;40(7):675-681.

6. Leung EW, Medeiros FA, Weinreb RN. Prevalence of ocular surface disease in glaucoma patients. J Glaucoma. 2008;17(5):350-355.

7. Garcia-Feijoo J, Sampaolesi JR. A multicenter evaluation of ocular surface disease prevalence in patients with glaucoma. Clin Ophthalmol. 2012;6:441-446.

8. Anwar Z, Wellik SR, Galor A. Glaucoma therapy and ocular surface disease: current literature and recommendations. Curr Opin Ophthalmol. 2013;24(2):136-143.

9. Stewart WC, Stewart JA, Nelson LA. Ocular surface disease in patients with ocular hypertension and glaucoma. Curr Eye Res. 2011; 36(5):391-398.

10. Herreras JM, Pastor JC, Calonge M, Asensio VM. Ocular surface alteration after long-term treatment with an anti-glaucomatous drug. Ophthalmology. 1992;99(7):1082-1088.

11. Arici MK, Arici DS, Topalkara A, Guler C. Adverse effects of topical anti-glaucoma drugs on the ocular surface. Clin Experiment Ophthalmol. 2000;28:113-117.

12. Aihara M, Otani S, Kozaki J, et al. Long-term effect of BAK-free travoprost on ocular surface and intraocular pressure in glaucoma patients after transition from latanoprost. J Glaucoma. 2012;21(1):60-64.

13. Thygesen J, Aaen K, Theodorsen F, Kessing SV, Prause JU. Shortterm effect of latanoprost and timolol eye drops on tear fluid and the ocular surface in patients with primary open-angle glaucoma and ocular hypertension. Acta Ophthalmol Scand. 2000;78(1):37-41.

14. Watson P, Stjernschantz J. A six-month, randomized, double-masked study comparing latanoprost with timolol in open-angle glaucoma and ocular hypertension. The Latanoprost Study Group. Ophthalmology. 1996;103(1):126-137.

15. Camras CB. Comparison of latanoprost and timolol in patients with ocular hypertension and glaucoma: a six-month masked, multicenter trial in the United States. The United States Latanoprost Study Group. Ophthalmology. 1996;103(1):138-147.

16. Kuppens EV, Stolwijk TR, de Keizer RJ, van Best JA. Basal tear turnover and topical timolol in glaucoma patients and healthy controls by fluorophotometry. Invest Ophthalmol Vis Sci. 1992;33(12):3442-3448.

17. Van Buskirk EM. Corneal anesthesia after timolol maleate therapy. Am J Ophthalmol. 1979;88(4):739-743.

18. Van Buskirk EM. Adverse reactions from timolol administration. Ophthalmology. 1980;87(5):447-450.

19. Wilson RP, Spaeth GL, Poryzees E. The place of timolol in the practice of ophthalmology. Ophthalmology. 1980;87(5):451-454.

20. Alm A, Stjernschantz J. Effects on intraocular pressure and side effects of $0.005 \%$ latanoprost applied once daily, evening or morning. A comparison with timolol. Scandinavian Latanoprost Study Group. Ophthalmology. 1995;102(12):1743-1752.

21. Sherwood MB, Grierson I, Millar L, Hitchings RA. Long-term morphologic effects of anti-glaucoma drugs on the conjunctiva and Tenon's capsule in glaucoma patients. Ophthalmology. 1989;96(3):327-335.

Clinical Ophthalmology

\section{Publish your work in this journal}

Clinical Ophthalmology is an international, peer-reviewed journal covering all subspecialties within ophthalmology. Key topics include: Optometry; Visual science; Pharmacology and drug therapy in eye diseases; Basic Sciences; Primary and Secondary eye care; Patient Safety and Quality of Care Improvements. This journal is indexed on Submit your manuscript here: http://www.dovepress.com/clinical-ophthalmology-journal
22. Brandt JD, Wittpenn JR, Katz LJ, Steinmann WN, Spaeth GL. Conjunctival impression cytology in patients with glaucoma using long-term topical medication. Am J Ophthalmol. 1991;112(3):297-301.

23. Baudouin C, Garcher C, Haouat N, Bron A, Gastaud P. Expression of inflammatory membrane markers by conjunctival cells in chronically treated patients with glaucoma. Ophthalmology. 1994;101(3): 454-460.

24. Baudouin C, Liang H, Hamard $\mathrm{P}$, et al. The ocular surface of glaucoma patients treated over the long term expresses inflammatory markers related to both T-helper 1 and T-helper 2 pathways. Ophthalmology. 2008;115(1):109-115.

25. Mirza SK, Higginbotham EJ. Iatrogenic glaucoma therapy failure: the adverse effects of topical glaucoma medication treatment outcome. Expert Rev Clin Pharmacol. 2009;2(1):87-99.

26. Lee SY, Wong TT, Chua J, Boo C, Soh YF, Tong L. Effect of chronic anti-glaucoma medications and trabeculectomy on tear osmolarity. Eye (Lond). 2013;27(10):1142-1150.

27. Neves Mendes CR, Hida RY, Kasahara N. Ocular surface changes in eyes with glaucoma filtering blebs. Curr Eye Res. 2012;37(4):309-311.

28. Lam J, Wong TT, Tong L. Ocular surface disease in posttrabeculectomy/ mitomycin C patients. Clin Ophthalmol. 2015;9:187-191.

29. The definition and classification of dry eye disease: report of the Definition and Classification Subcommittee of the International Dry Eye Work Shop (2007). Ocul Surf. 2007;5(2):75-92.

30. Fuentes-Paez G, Herreras JM, Cordero Y, Almaraz A, Gonzalez MJ, Calonge M. [Lack of concordance between dry eye syndrome questionnaires and diagnostic tests]. Arch Soc Esp Oftalmol. 2011;86(1):3-7. Spanish.

31. Moore JE, Graham JE, Goodall EA, et al. Concordance between common dry eye diagnostic tests. Br J Ophthalmol. 2009;93(1):66-72.

32. Chalmers RL, Begley CG, Edrington T, et al. The agreement between self-assessment and clinician assessment of dry eye severity. Cornea. 2005;24(7):804-810.

33. Versura P, Cellini M, Torreggiani A, Profazio V, Bernabini B, Caramazza R. Dryness symptoms, diagnostic protocol and therapeutic management: a report on 1,200 patients. Ophthalmic Res. 2001; 33(4):221-227.

34. Malet F, Le Goff M, Colin J, et al. Dry eye disease in French elderly subjects: the Alienor study. Acta Ophthalmol. 2014;92(6):e429-e436.

35. Saade CE, Lari HB, Berezina TL, Fechtner RD, Khouri AS. Topical glaucoma therapy and ocular surface disease: a prospective controlled cohort study. Can J Ophthalmol. 2015;50(2):132-136.

36. Ono T, Yuki K, Ozeki N, Shiba D, Tsubota K. Ocular surface complications after trabeculectomy: incidence, risk factors, time course and prognosis. Ophthalmologica. 2013;230(2):93-99.

37. Schwartz GS, Holland EJ. Iatrogenic limbal stem cell deficiency: when glaucoma management contributes to corneal disease. J Glaucoma. 2001;10(6):443-445

38. Rolando M, Zierhut M. The ocular surface and tear film and their dysfunction in dry eye disease. Surv Ophthalmol. 2001;45(Suppl 2): S203-S210

39. Varma R, Lee PP, Goldberg I, Kotak S. An assessment of the health and economic burdens of glaucoma. Am J Ophthalmol. 2011;152(4): $515-522$.

40. Rein DB, Zhang P, Wirth KE, et al. The economic impact of major visual disorders in the United States. Arch Ophthalmol. 2006;124(12):1754-1760.
PubMed Central and CAS, and is the official journal of The Society of Clinical Ophthalmology (SCO). The manuscript management system is completely online and includes a very quick and fair peer-review system, which is all easy to use. Visit http://www.dovepress.com/ testimonials.php to read real quotes from published authors. 